\title{
Red and processed meat consumption and risk of bladder cancer: a dose-response meta-analysis of epidemiological studies
}

\author{
Alessio Crippa $^{1} \cdot$ Susanna C. Larsson $^{3} \cdot$ Andrea Discacciati $^{2} \cdot$ Alicja Wolk $^{3}$ • \\ Nicola Orsini ${ }^{1}$
}

Received: 28 July 2016 / Accepted: 30 December 2016 / Published online: 22 December 2016

(C) The Author(s) 2016. This article is published with open access at Springerlink.com

\begin{abstract}
Background/objectives Several epidemiological studies have analyzed the associations between red and processed meat and bladder cancer risk but the shape and strength of the associations are still unclear. Therefore, we conducted a dose-response meta-analysis to quantify the potential association between red and processed meat and bladder cancer risk.

Methods Relevant studies were identified by searching the PubMed database through January 2016 and reviewing the reference lists of the retrieved articles. Results were combined using random-effects models.

Results Five cohort studies with 3262 cases and 1,038,787 participants and 8 cases-control studies with 7009 cases and 27,240 participants met the inclusion criteria. Red meat was linearly associated with bladder cancer risk in casecontrol studies, with a pooled RR of 1.51 (95\% confidence interval (CI) $1.13,2.02$ ) for every $100 \mathrm{~g}$ increase per day, while no association was observed among cohort studies $(P$ heterogeneity across study design $=0.02)$. Based on
\end{abstract}

Electronic supplementary material The online version of this article (doi:10.1007/s00394-016-1356-0) contains supplementary material, which is available to authorized users.

Alessio Crippa

alessio.crippa@ki.se

1 Public Health Sciences, Karolinska Institutet, Tomtebodavagen 18A, 17177 Stockholm, Sweden

2 Unit of Biostatistics, Institute of Environmental Medicine, Karolinska Institutet, Nobels Vag 13, 17177 Stockholm, Sweden

3 Unit of Nutritional Epidemiology, Institute of Environmental Medicine, Karolinska Institutet, Nobels Vag 13, 171

77 Stockholm, Sweden both case-control and cohort studies, the pooled relative risk (RR) for every $50 \mathrm{~g}$ increase of processed meat per day was $1.20(95 \%$ CI $1.06,1.37)(P$ heterogeneity across study design $=0.22$ ).

Conclusions This meta-analysis suggests that processed meat may be positively associated with bladder cancer risk. A positive association between red meat and risk of bladder cancer was observed only in case-control studies, while no association was observe in prospective studies.

Keywords Red meat · Processed meat $\cdot$ Bladder cancer . Dose-response $\cdot$ Meta-analysis

\section{Introduction}

Bladder cancer is the fifth most common cancer among men and the fourteenth among women with an estimated number of 429,000 cases worldwide in 2012 [1]. Bladder cancer is rather common in developed countries (North America and Europe), and it is more frequent among persons aged 75 or older [2]. Mortality rates have been stable over the last decade with 165,000 estimated deaths in 2012 [1]. A few risk factors have recently been linked to the etiology of bladder cancer. Apart from age and gender, cigarette smoking and specific occupational exposures are considered the most important risk factors [3, 4]. Identification of additional modifiable risk factors such as diet may enhance primary prevention.

Recently two meta-analyses summarized the body of evidence concerning red and processed meat consumption and risk of bladder cancer [5, 6]. Results from the review by Wang et al. [5] indicated an increased risk of bladder cancer of 17 and $10 \%$ for high red meat and high processed meat consumption, respectively. The more recent review by 
Li et al. [6], on the other hand, found a significant association for processed meat, with a $22 \%$ increased risk of bladder cancer for high consumption but not for red meat consumption. Both meta-analyses, however, were based only on contrasting risk estimates for the highest vs. the lowest category of meat consumption, and this has some limitations when the exposure distribution vary substantially across studies. In the review by Li et al. [6], one of the included studies [7] conducted in Uruguay, for instance, used $0-150 \mathrm{~g} / \mathrm{day}$ of red meat consumption (median of $85 \mathrm{~g} /$ day) as the lowest category. In another study conducted in the USA [8], >58.5 g/day was the highest category for red meat consumption.

Our aim is to describe variation in bladder cancer risk across the whole range of the exposure distribution. A dose-response analysis is more efficient and less sensitive to heterogeneity of the exposure across different study populations. Therefore, we conducted a dose-response metaanalysis to clarify and quantify the potential association between red and processed meat and bladder cancer risk.

\section{Materials and methods}

\section{Literature search and selection}

Eligible studies were identified by searching the PubMed database through July 2016, with the terms ["bladder" AND ("carcinoma" or "cancer" or "tumor" OR "neoplasms")] AND ("meat" or "beef" or "pork" or "lamb"). In addition, the reference lists of the retrieved articles were examined to identify additional reports. The search was restricted to studies written in English and carried out in human. We performed this meta-analysis accordingly to the Meta-Analysis of Observational Studies in Epidemiology (MOOSE) guidelines [9]. Two authors (A.C. and A.D.) independently retrieved the data from studies on the association between red and processed meat and risk of bladder cancer. Discrepancies were discussed and resolved.

Studies were eligible if they met the following criteria: (1) the study was a cohort or case-control study; (2) the exposure of interest was red meat and/or processed meat; (3) the outcome was incidence of bladder cancer; (4) the authors reported measures of association (hazard ratio, relative risk, odds ratio) with the corresponding confidence intervals for three or more categories for red or processed meat consumption. In case of multiple reports on the same study population, we included only the most comprehensive or recent one.

\section{Data extraction}

From each study, we extracted the following information: first author's surname, year of publication, study design, country where the study was conducted, study period, exposure definition, unit of measurement, number of cases, study size, confounding variables, and measure of associations for all the categories of meat consumption together with their confidence intervals. Given the low prevalence of bladder cancer, the odds ratios were assumed approximately the same as the relative risks (RRs). When several risk estimates were available, we included those reflecting the greatest degree of adjustment.

\section{Statistical analysis}

We used the method described by Greenland and Longnecker [10] and Orsini et al. [11] to reconstruct study-specific trend from aggregated data, taking into accounts the covariance among the log RR estimates. Risk estimates from studies not reporting information about the number of deaths and study size did not allow reconstruction of the covariance and were assumed independent. Potential nonlinear associations were assessed by use of restricted cubic splines with three knots located at the 10th, 50th, and 90th percentiles of the exposure distribution. An overall $P$ value was calculated by testing that the regression coefficients were simultaneously equal to zero. A $P$ value for nonlinearity was obtained by testing that the coefficient of the second spline term was equal to zero [12].

Since studies used different units to express meat consumption (e.g., servings/day, grams/day, grams per $1000 \mathrm{kcal} /$ day), we converted frequency of consumption using 120 and $50 \mathrm{~g}$ as the average portion sizes for red and processed meat, respectively. We chose those values in accordance with previous meta-analyses on meat consumption and other types of cancer $[13,14]$ and results from both the Continuing Survey of Food Intakes by Individuals [15] and the European Prospective Investigation into Cancer and Nutrition [16]. Meat consumption reported in grams per $1000 \mathrm{kcal} / \mathrm{day}$ was converted to $\mathrm{g} /$ day using the average energy intake reported in the original articles. Within each exposure category, the median or mean value was assigned to the corresponding RRs. If not reported, we assigned the midpoint of the upper and lower boundaries as average consumption. If the upper bound of the highest category was not reported, we assumed that the category had the same width as the contiguous one. When RRs were reported only for single food items (e.g., separately for beef and pork), we combined them using a fixed-effects model and used the pool estimate as summary measure.

A random-effects meta-analysis was adopted to acknowledge heterogeneity across study findings. Statistical heterogeneity was further assessed by using the $Q$ test (defined as a $P$ value less than 0.10 ) and quantified by $R_{b}$ statistic [17]. Meta-regression models were employed to explain residual heterogeneity. Differences in doseresponse curves between subgroups of studies were tested 
as described by Berlin et al. [18]. Evaluation of goodnessof-fit for the final models was assessed using the set of tools presented by Discacciati et al. [19]. Publication bias was investigated using the Egger asymmetry test [20].

We performed sensitivity analyses (1) excluding studies where red meat definition included also some items of processed meat; (2) excluding studies that did not adjust for energy intake; (3) evaluating alternative average portion sizes for red meat (100 and $140 \mathrm{~g}$ ) and processed meat (30 and $70 \mathrm{~g}$ ) consumption. All statistical analyses were conducted with the dosresmeta [21] and metafor [22] packages in R (R Foundation for Statistical Computing, Vienna, Austria) [23]. $P$ values less than 0.05 were considered statistically significant.

\section{Results}

\section{Literature search}

The search strategy identified 146 articles, 108 of which were excluded after review of the title or abstract (Fig. 1).
Of the 38 eligible papers 14 were excluded because they did not meet the inclusion criteria (not original articles, outcome different from bladder cancer, or not reporting risk estimates with their confidence intervals). The reference lists of the remaining 24 articles were checked for additional pertinent reports, and 5 additional papers were identified. We further excluded 16 additional articles: 8 presented duplicated publications [24-31]; 3 analyzed bladder and other urinary cancer together [32-34]; 3 did not report enough data for a dose-response analysis [35-37]; and 2 did not report results for red or processed meat consumption [16, 38]. Thus, the meta-analysis included 13 independent epidemiological studies [7, 8, 31, 39-49].

\section{Study characteristics}

The main characteristics of the 13 epidemiological studies included in the meta-analysis are presented in Table 1. Five cohort studies [39-43] with 3262 cases and 1038,787 participants and 8 cases-control studies, of which 4 population-based [8, 44, 46, 47] and 4 hospital-based [7, 45, 48, 49], with 7009 cases and 27,240 participants evaluated
Fig. 1 Selection of studies for inclusion in a meta-analysis of red and processed meat consumption and risk of bladder cancer 1966-2016

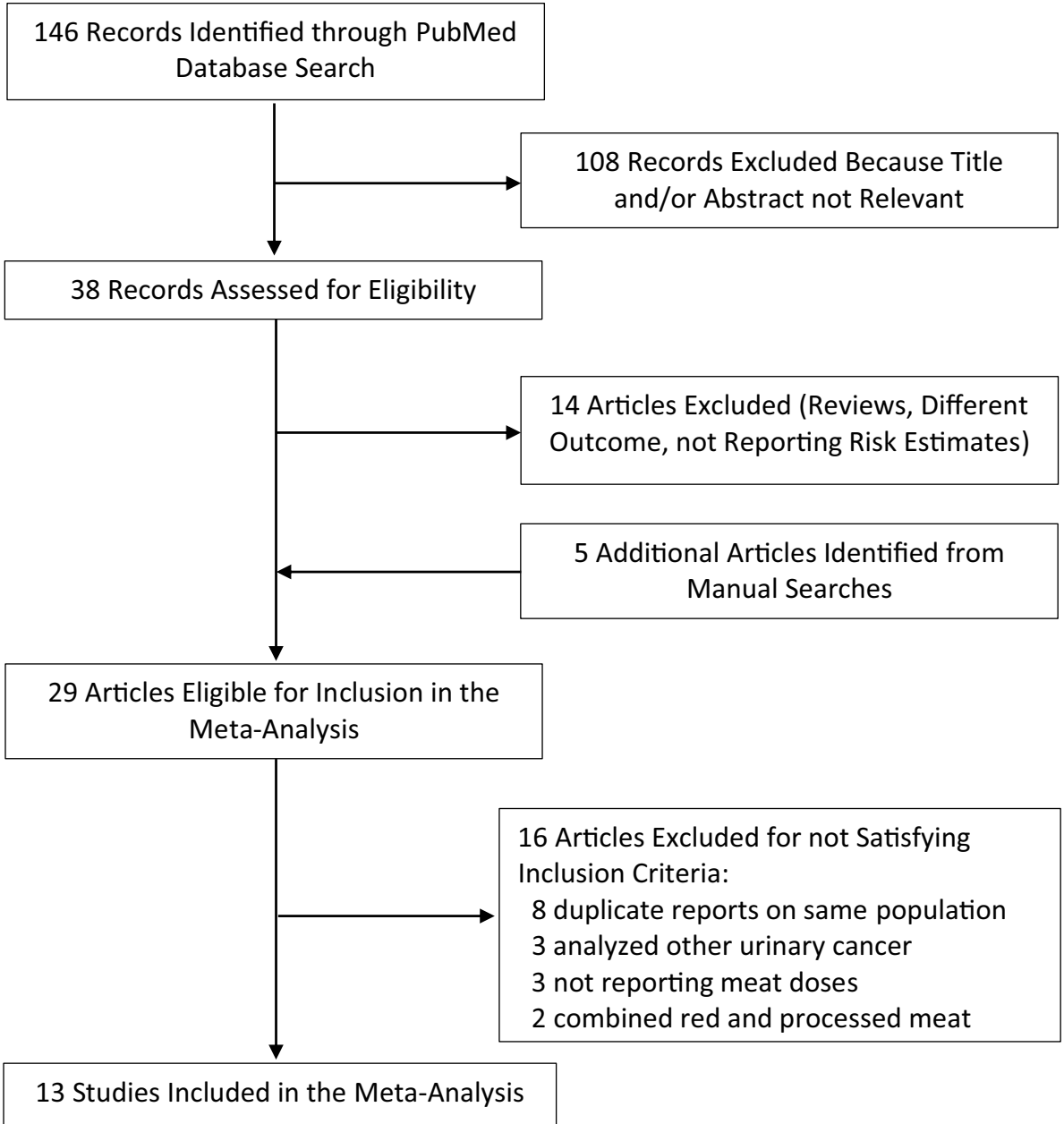




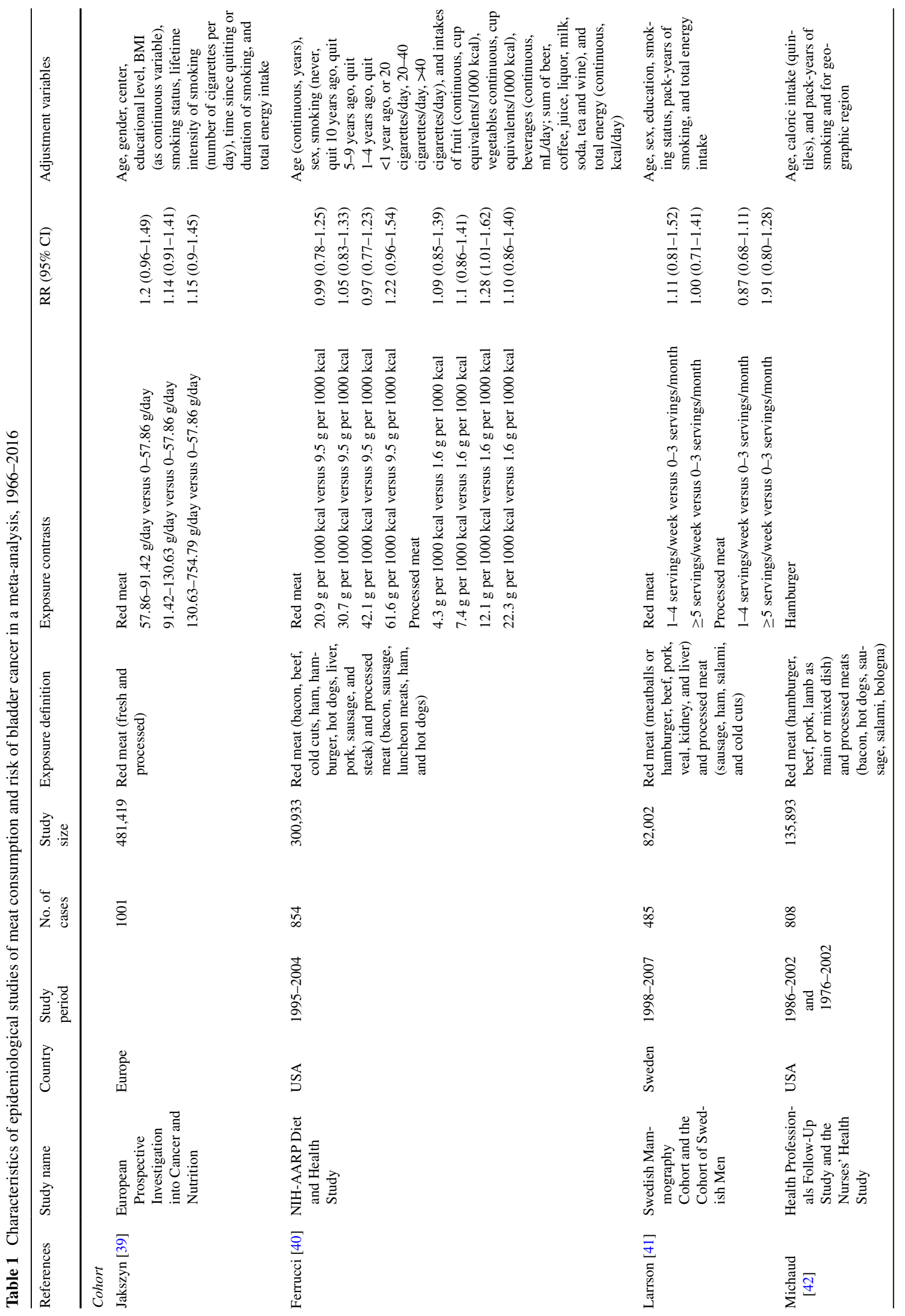




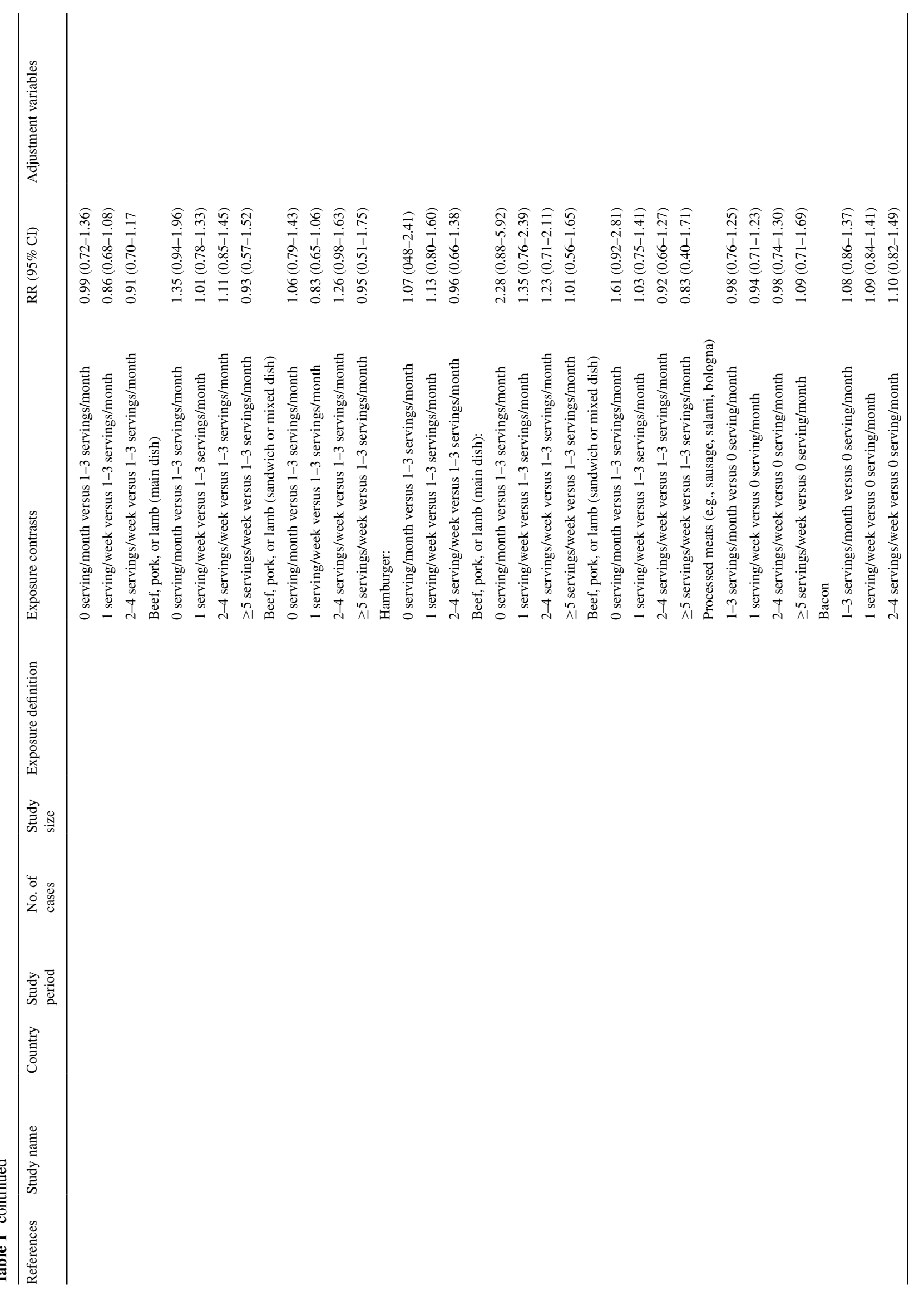




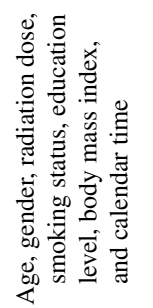

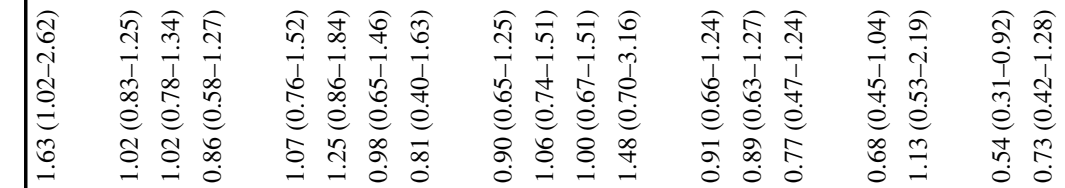

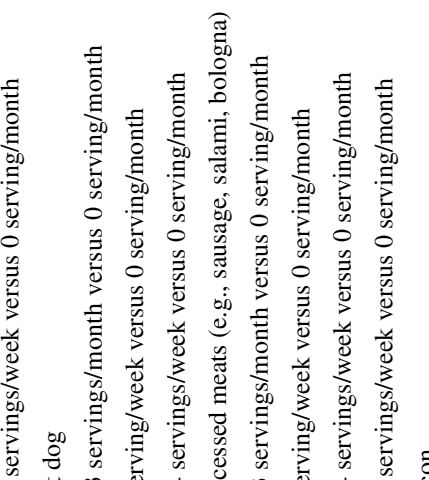

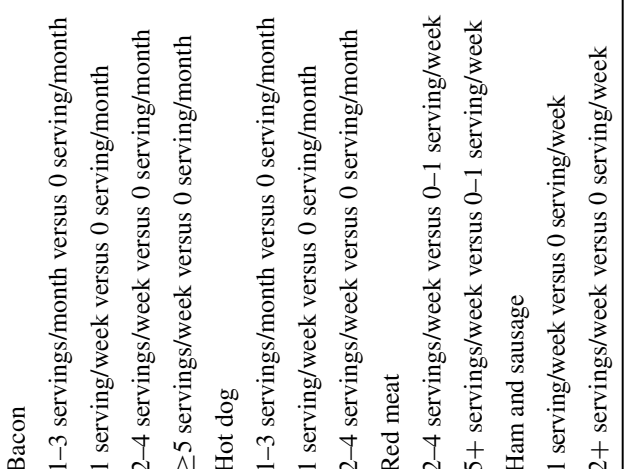

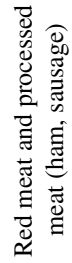

早

$\stackrel{+}{=}$

$\frac{\substack{2 \\ \frac{1}{2}}}{2}$

喜

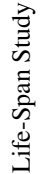

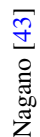




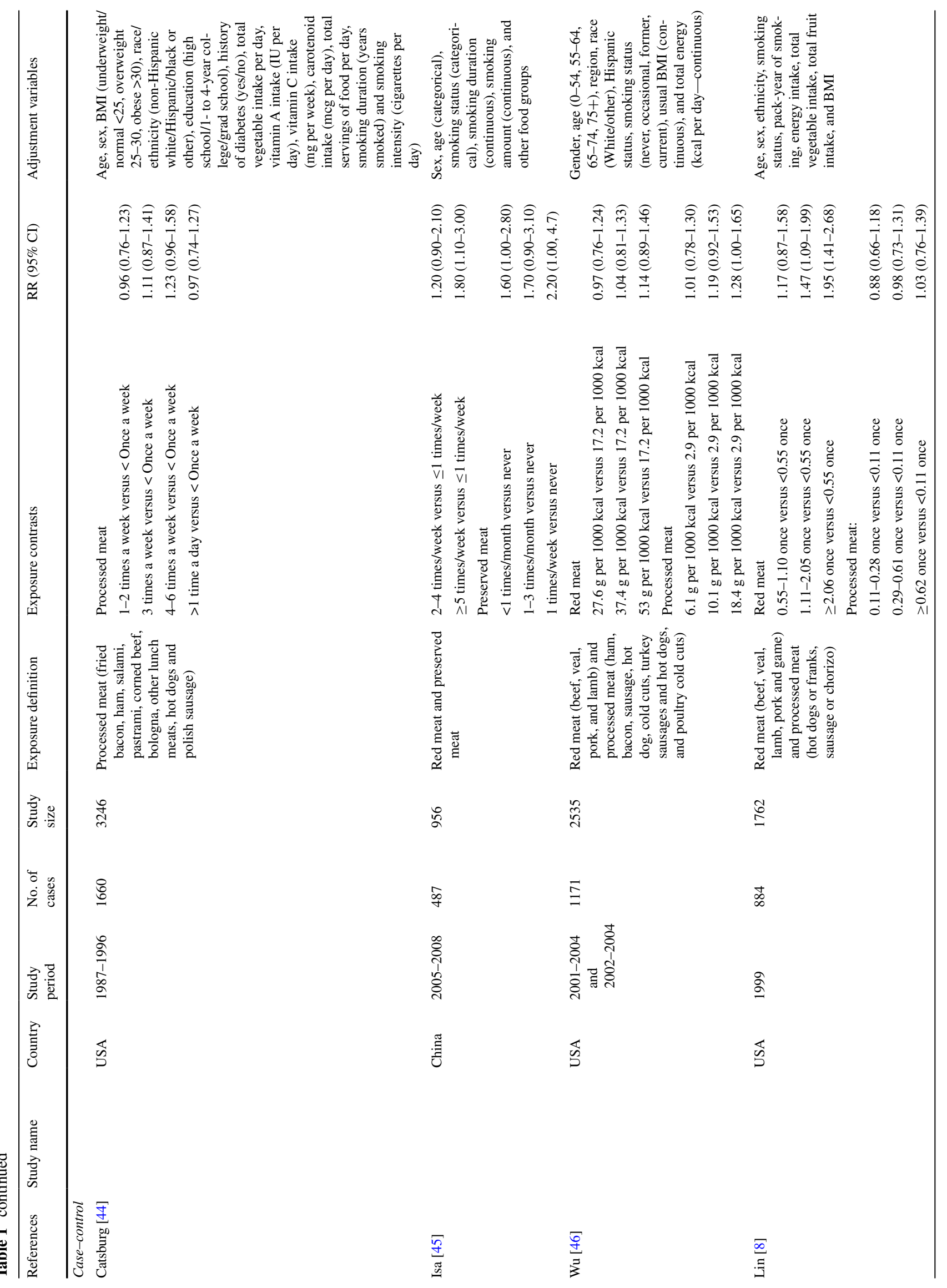




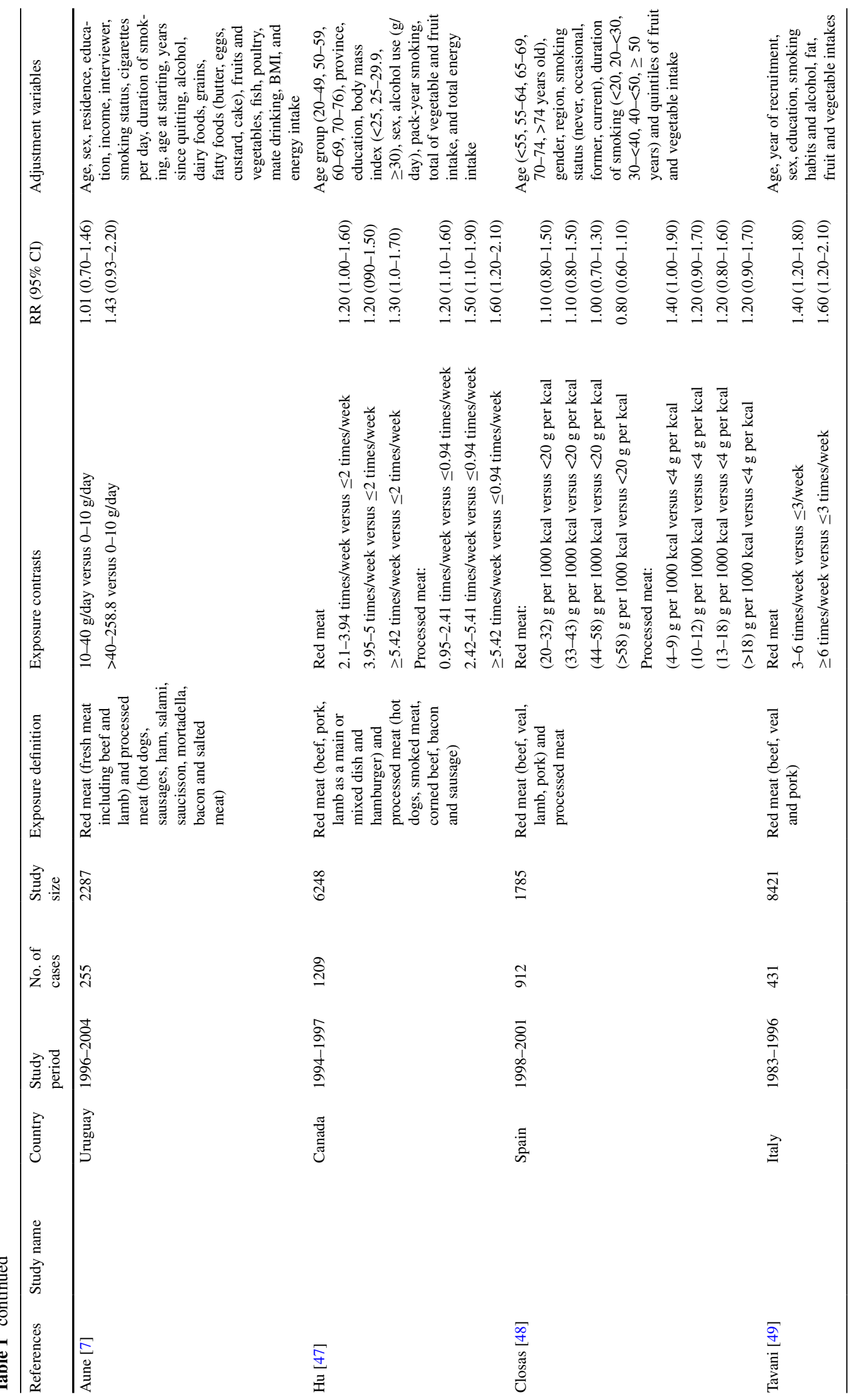


the relation between red and/or processed meat and risk of bladder cancer. Two articles [39, 49] reported results only for red meat, while one [44] only for processed meat. Definition of meat and red meat varied across studies but generally included beef, veal, pork, and lamb for red meat, and bacon, ham, salami, sausages, and hot dogs for processed meat. Two cohort studies [39, 40] included also processed meat in the definition of red meat, and one study [42] included only results for specific food items. One study [44] reported results only for liver intake and was not included in the analysis of red meat. Another study [45] analyzed preserved meat consumption and, given the limited range of exposure (up to 1/week), was excluded from the analysis of processed meat.

Only 3 studies [40, 46, 48] considered different cooking methods and doneness levels for meat consumption. None of them found evidence of an association between preparation methods and bladder cancer. Different units were used to report meat consumption: servings/week (7 studies), grams per $1000 \mathrm{kcal}$ per day (3 studies), and grams per day ( 3 studies). Five studies were conducted in the USA, 4 in Europe, and 1 each in Canada, Uruguay, China, and Japan. All the studies were carried out in both men and women, and only one study [42] reported results separately by gender. All the studies provided measure of associations adjusted for age, gender, and smoking. Four studies did not adjust for energy intake [43-45, 49]. Other common adjusting variables were other food groups (8 studies), BMI (6 studies), education (6 studies). Additional covariates were less consistent across studies.

\section{Association between red meat consumption and risk of bladder cancer}

We found a statistically significant association between red meat consumption and risk of bladder cancer $(P=0.009$; $P$ nonlinearity $=0.74)$ (Online Resource 1$)$. The summary $\mathrm{RR}$ for an increment of $100 \mathrm{~g}$ per day of red meat was 1.22 (95\% CI 1.05, 1.41). There was substantial between-studies heterogeneity $\left(R_{b}=67 \%, P<0.01\right)$. Egger's regression test did not suggest the presence of substantial publication bias $(P=0.14)$.

There was statistical heterogeneity according to study design $(P$ for heterogeneity $=0.02)$. The pooled RR restricted to the cohort studies was 1.01 (95\% CI 0.97 , 1.06) for an increment of $100 \mathrm{~g}$ per day of red meat with no significant heterogeneity $\left(R_{b}=0 \%, P=0.62\right)$ (Figure 2). The deviance test did not detect lack of fit $(D=24$, $d f=18, P=0.17)$. In case-control studies, the corresponding pooled RR was $1.51(95 \%$ CI $1.13,2.02)$ with substantial heterogeneity among studies $\left(R_{b}=81 \%\right.$,
$P<0.01)$ and overall indication of poor fit $(D=44$, $d f=18, P<0.01)$.

No differences were found according to study location $(P$ for heterogeneity $=0.7)$, units of measurement $(P$ for heterogeneity $=0.38$ ), and selection of controls ( $P$ for heterogeneity $=0.65$ ). Excluding those studies with also processed meat in the definition of red meat, the pooled RRs were 1.27 (95\% CI 1.03, 1.57) overall and 0.95 (95\% CI $0.82,1.11)$ restricted to cohort studies. The pooled $\mathrm{RR}$ for an increment of $100 \mathrm{~g}$ of red meat per day was $1.14(95 \%$ CI $0.99,1.31)$ based on studies that adjusted for energy intake. In the sensitivity analysis for alternative average portion sizes of red meat, the results did not substantially change. The pooled RR for an increment of $100 \mathrm{~g}$ of red meat per day was 1.27 and 1.19 for assigned portions of $140 \mathrm{~g}$ per day and $100 \mathrm{~g}$ per day, respectively.

For an increment of four servings per week of red meat (120 g per servings), the summary RR of bladder cancer was 1.15 (95\% CI 1.03, 1.27) overall, 1.01 (95\% CI 0.98, 1.04) for cohort studies, and $1.32(95 \%$ CI $1.08,1.62)$ for case-control studies.

\section{Association between processed meat consumption and risk of bladder cancer}

We found a statistically significant association between processed meat intake and bladder cancer with no departure from linearity $(P=0.005, P$ nonlinearity $=0.92)$ (Online Resource 2). Every $50 \mathrm{~g}$ increase in processed meat per week was associated with a $20 \%(95 \%$ CI 6,37$)$ increase in risk of bladder cancer with moderate heterogeneity $\left(R_{b}=38 \%\right.$, $P=0.07)$. Egger's regression test did not detect publication bias $(P=0.21)$. No evidence of lack of fit was observed $(D=43, d f=34, P=0.14)$. The test did not detect significant differences between case-control and cohort studies $(P$ for heterogeneity $=0.22$ ). Stratified analysis provided a RR of $1.10(95 \%$ CI $0.95,1.27)$ and 1.31 (95\% CI 1.06, 1.63) for cohort and case-control studies, respectively (Fig. 3).

The associations were similar across strata of study location $(P$ for heterogeneity $=0.68)$, units of measurement $(P$ for heterogeneity $=0.71)$, and selection of controls $(P$ for heterogeneity $=0.46)$. Exclusion of studies that did not adjust for energy intake provided a pooled RR of 1.24 (95\% CI 1.07, 1.43). Similar results were observed for alternative average portion sizes of $30 \mathrm{~g}$ per day and $70 \mathrm{~g}$ per day with pooled RR, respectively, of 1.14 and 1.36 for an increment of $50 \mathrm{~g}$ per day of processed meat.

For an increment of four servings per week of processed meat ( $50 \mathrm{~g}$ per servings), the summary RR of bladder cancer was $1.11(95 \%$ CI 1.03, 1.20) overall, 1.06 (95\% CI $0.97,1.15)$ for cohort studies, and 1.17 (95\% CI 1.03, 1.32) for case-control studies. 


\section{Red meat and bladder cancer \\ for every $100 \mathrm{~g}$ per day increment}

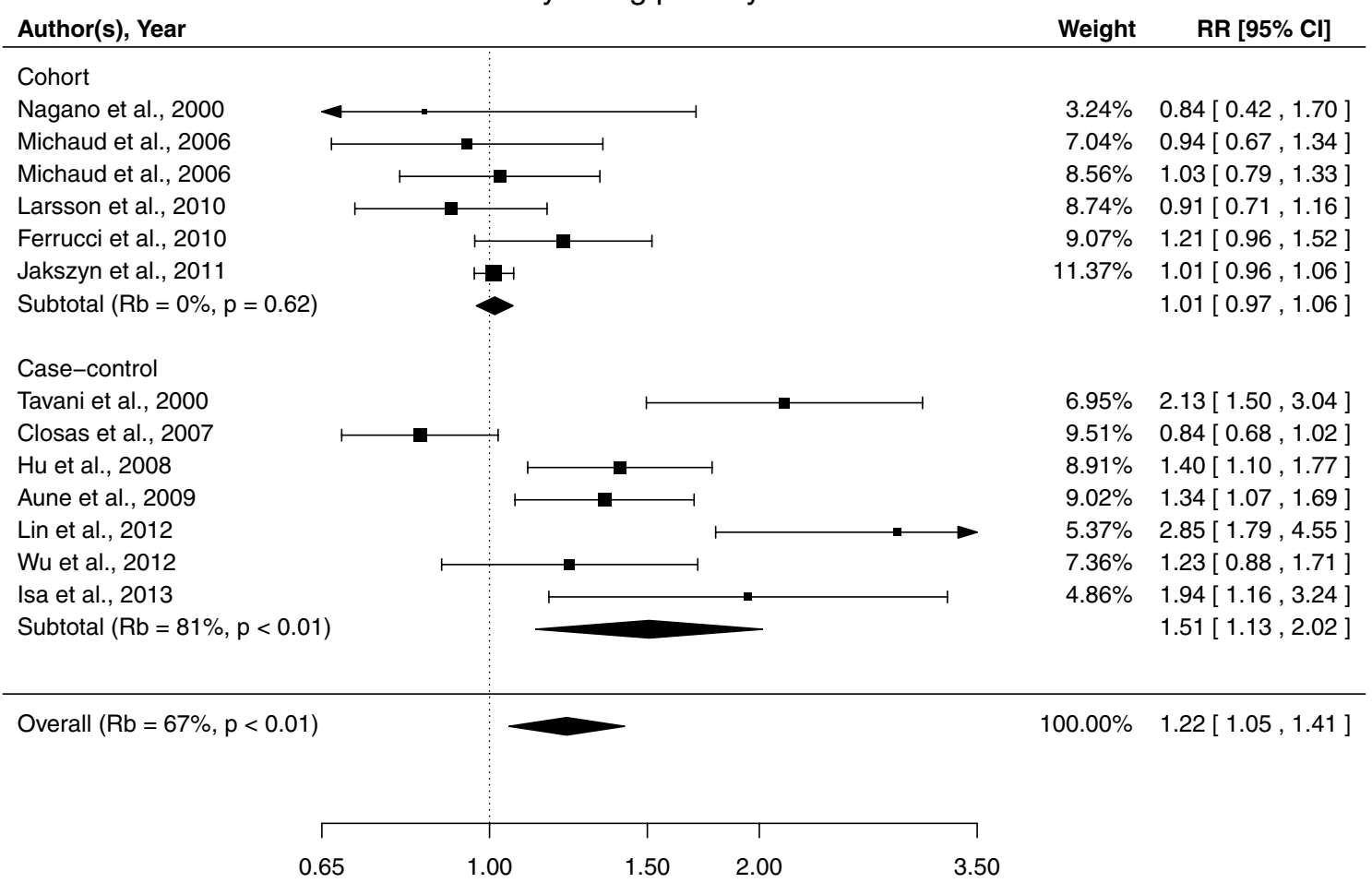

Fig. 2 Relative risks of bladder cancer with $100 \mathrm{~g}$ per day increment in red meat consumption separately for cohort and case-control studies

\section{Discussion}

Findings from this dose-response meta-analysis of five cohort and eight case-control studies suggest that processed meat consumption is positively associated with risk of bladder cancer. An increment of $50 \mathrm{~g}$ of processed meat per day was associated with $20 \%$ increased risk of bladder cancer. Red meat consumption was associated with bladder cancer only in case-control studies, with a $51 \%$ increased risk of an increment of $100 \mathrm{~g}$ per day, while no association was observed among the prospective studies.

Meat, in particular processed meat, is a potential risk factor for several cancers, with the most convincing evidence for colorectal cancer [50]. In 2015, the International Agency for Research on Cancer classified processed meats as carcinogenic to humans (Group 1) and red meat as probably carcinogenic to humans [51]. The contribution of meat to the etiology of bladder cancer may be explained by different mechanisms, given that many nutrients are excreted through the urinary tract [52]. The most established mechanism involves the formation of endogenous nitrosamines from nitrites that are particularly abundant in processed meats [53]. Experimental studies have shown that some nitrosamine metabolites induce bladder tumors in rodents $[54,55]$. Further support for at potential role of nitrosamines in bladder carcinogenesis is that cigarette smoking is a strong risk factor for bladder cancer and tobacco smoke is a main source of exogenous exposure to nitrosamines. Consumption of red meat could potentially increase the risk of bladder cancer through heterocyclic amines and polycyclic aromatic hydrocarbons, which can be generated from high temperature cooking [56]. Heterocyclic amines and polycyclic aromatic hydrocarbons have been consistently shown to be carcinogenic in animal studies $[56,57]$.

A direct comparison with the results of previous reviews $[5,6]$ is difficult since they were based on study-specific risk estimates for high versus low categories of meat consumption, which varied substantially across studies. The directions of the associations, however, were consistent, even though an association was found only for processed meat in the meta-analysis by Lin et al. [6]. As in the review by Wang et al. [5], case-control studies provided stronger risk estimates as compared to prospective studies.

Among several potential explanations, case-control studies generally assess the exposure after diagnosis, and therefore, recall bias may lead to differential misclassification between cases and controls. Considering the limited knowledge of the role of meat consumption on the development of bladder cancer [44], such classification errors are 
processed meat and bladder cancer

for every $50 \mathrm{~g}$ per day increment

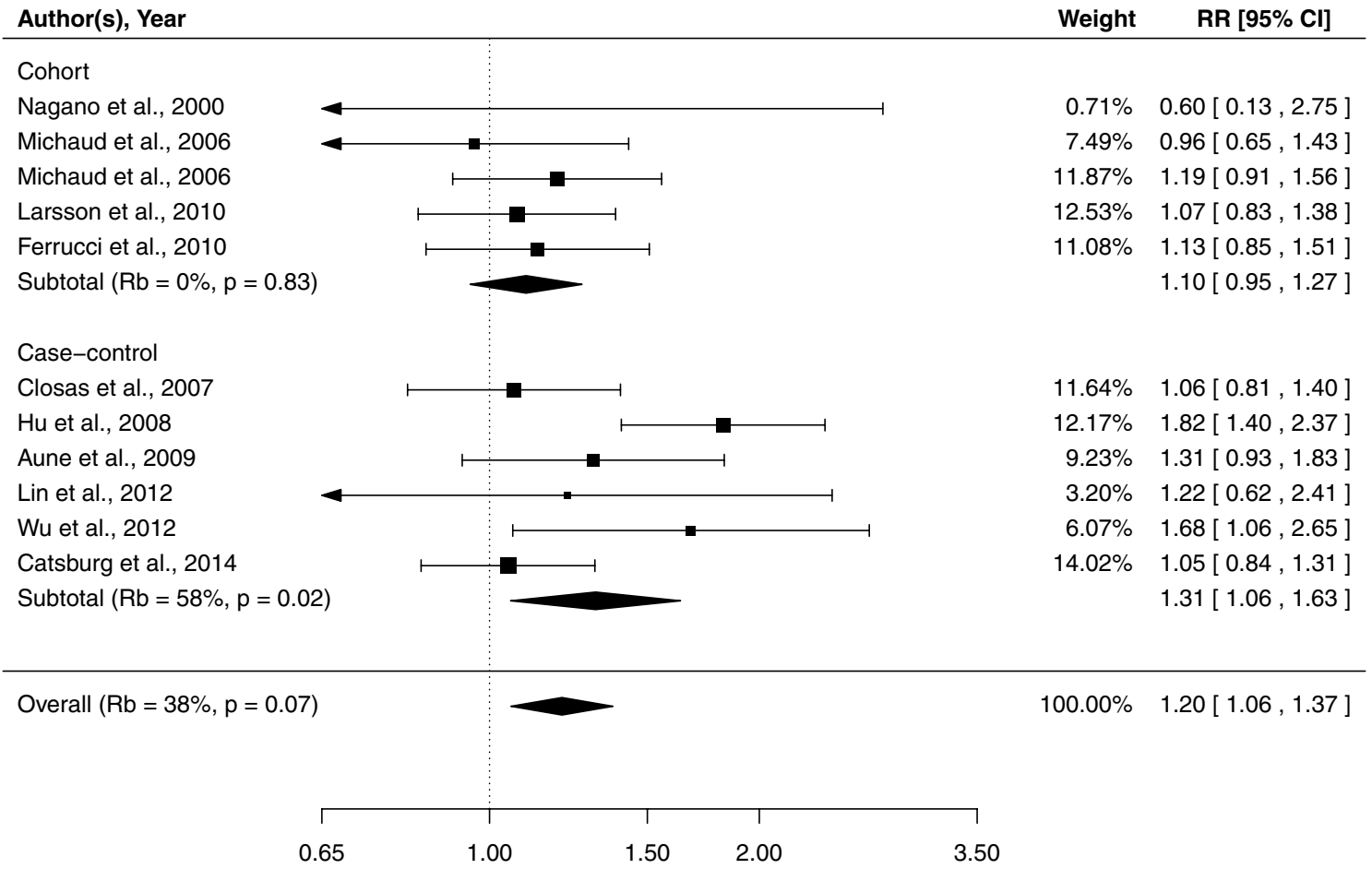

Fig. 3 Relative risks of bladder cancer with $50 \mathrm{~g}$ per day increment in processed meat consumption separately for cohort and case-control studies

likely to be similar among cases and controls. On the other hand, half of the control studies used hospital-based controls which may inflate the pooled association in case controls have been recruited for conditions linked with changes in meat consumption. Although based on limited number of studies, we did not observed differences in results between hospital-based and population-based case-control studies. Different participation rates related to exposure or severity of diseases may also be a selection bias among casecontrol studies. In addition, the time between diagnosis and the exposure assessment is generally shorter for casecontrol studies; hence, it may not reflect long-term exposure because of changes in dietary patterns. On the other hand, in cohort studies participants may alter their dietary intake during the follow-up, which may bias results toward the null hypothesis of no association. One of the included cohort studies [42] analyzed repeated dietary measurements over time and observed stronger associations when using cumulative update date and when removing participant who had change their meat consumption.

Strength of this review is the dose-response analysis, which better takes into account the quantitative nature and heterogeneity of the exposure. In our analysis, all the information about meat consumption, including intermediate categories, contributed to the pooled associations. Another strength is the large number of cases that provided high statistical power to detect associations of moderate magnitude. Lastly, no evidence of publication bias was observed.

This meta-analysis also had potential limitations. Pooling results from epidemiological studies do not solve the problem of residual confounding, which inherently affects individual studies. All of the included studies, however, adjusted for main known risk factors for bladder cancer such as age, gender, and smoking, and some studies also adjusted for energy intake, BMI, education, and other food groups. Excluding those studies not adjusting for energy intake did not change the overall results, suggesting that energy intake may have a limited impact on developing bladder cancer. Second, red and processed meat definition varied across study and this may partially contribute to the observed heterogeneity. Different units of measurements were also used to report risk estimates for meat consumption, and we had to assume average portion sizes when meat consumption was reported as servings. Nevertheless, stratified analysis for different types of measurements and sensitivity analysis for alternative portion sizes did not find substantial differences in results. Third, it was not possible to investigate the association between different meat-cooking methods and bladder 
cancer because only three articles reported such information. However, none of them found an increase in bladder cancer risk with any of the cooking methods. Fourth, statistical heterogeneity was observed in our analysis as in the previous two reviews $[5,6]$ but was mainly explained by different study design. After stratification, moderate heterogeneity was still observed among case-control studies, while cohort studies provided more homogenous results.

In conclusion, results from this dose-response metaanalysis suggest that processed meat consumption may be positively associated with risk of bladder cancer. Positive association between red meat and risk of bladder cancer was observed only in case-control studies, while no association was observed in prospective studies.

Acknowledgements This work was partly supported by Young Scholar Award from the Karolinska Institutet's Strategic Program in Epidemiology.

Authors' contribution All authors (AC, SL, AD, AW, and NO) participated both in the study design and in writing the manuscript. AC and $\mathrm{AD}$ participated in the data collection. $\mathrm{AC}$ analyzed the data and wrote the manuscript under the supervision of NO. SL and AW interpreted the results and critically reviewed the paper. All authors read and approved the final manuscript.

\section{Compliance with ethical standards}

Conflict of interest Authors declare that they have no conflict of interest.

Open Access This article is distributed under the terms of the Creative Commons Attribution 4.0 International License (http://creativecommons.org/licenses/by/4.0/), which permits unrestricted use, distribution, and reproduction in any medium, provided you give appropriate credit to the original author(s) and the source, provide a link to the Creative Commons license, and indicate if changes were made.

\section{References}

1. Ferlay J, Soerjomataram I, Dikshit R et al (2015) Cancer incidence and mortality worldwide: sources, methods and major patterns in GLOBOCAN 2012. Int J Cancer 136:E359-E386. doi:10.1002/ijc. 29210

2. Jemal A, Bray F, Center MM et al (2011) Global cancer statistics. CA Cancer J Clin 61:69-90. doi:10.3322/caac.20107

3. Murta-Nascimento C, Schmitz-Dräger BJ, Zeegers MP et al (2007) Epidemiology of urinary bladder cancer: from tumor development to patient's death. World J Urol 25:285-295. doi:10.1007/s00345-007-0168-5

4. Johansson SL, Cohen SM (1997) Epidemiology and etiology of bladder cancer. Semin Surg Oncol 13:291-298

5. Wang C, Jiang H (2011) Meat intake and risk of bladder cancer: a meta-analysis. Med Oncol 29:848-855. doi:10.1007/ s12032-011-9985-x

6. Li F, An S, Hou L et al (2014) Red and processed meat intake and risk of bladder cancer: a meta-analysis. Int J Clin Exp Med 7:2100-2110
7. Aune D, De Stefani E, Ronco A et al (2009) Meat consumption and cancer risk: a case-control study in Uruguay. Asian Pac J Cancer Prev APJCP 10:429-436

8. Lin J, Forman MR, Wang J et al (2012) Intake of red meat and heterocyclic amines, metabolic pathway genes and bladder cancer risk. Int J Cancer J Int Cancer 131:1892-1903. doi:10.1002/ ijc. 27437

9. Stroup DF, Berlin JA, Morton SC et al (2000) Meta-analysis of observational studies in epidemiology: a proposal for reporting. JAMA 283:2008-2012. doi:10.1001/jama.283.15.2008

10. Greenland S, Longnecker MP (1992) Methods for trend estimation from summarized dose-response data, with applications to meta-analysis. Am J Epidemiol 135:1301-1309

11. Orsini N, Bellocco R, Greenland S (2006) Generalized least squares for trend estimation of summarized dose-response data. Stata J 6:40-57

12. Desquilbet L, Mariotti F (2010) Dose-response analyses using restricted cubic spline functions in public health research. Stat Med 29:1037-1057. doi:10.1002/sim.3841

13. Xue X-J, Gao Q, Qiao J-H et al (2014) Red and processed meat consumption and the risk of lung cancer: a dose-response meta-analysis of 33 published studies. Int J Clin Exp Med 7:1542-1553

14. Norat T, Lukanova A, Ferrari P, Riboli E (2002) Meat consumption and colorectal cancer risk: dose-response meta-analysis of epidemiological studies. Int J Cancer 98:241-256. doi:10.1002/ ijc. 10126

15. Krebs-Smith SM, Cleveland LE, Ballard-Barbash R et al (1997) Characterizing food intake patterns of American adults. Am J Clin Nutr 65:1264S-1268S

16. Riboli E, Kaaks R (1997) The EPIC Project: rationale and study design. European Prospective Investigation into Cancer and Nutrition. Int J Epidemiol 26:S6. doi:10.1093/ije/26.suppl_1.S6

17. Crippa A, Khudyakov P, Wang M et al (2016) A new measure of between-studies heterogeneity in meta-analysis. Stat Med. doi:10.1002/sim.6980

18. Berlin JA, Longnecker MP, Greenland S (1993) Meta-analysis of epidemiologic dose-response data. Epidemiol Camb Mass 4:218-228

19. Discacciati A, Crippa A, Orsini N (2015) Goodness of fit tools for dose-response meta-analysis of binary outcomes. Res Synth Methods. doi:10.1002/jrsm.1194

20. Egger M, Davey Smith G, Schneider M, Minder C (1997) Bias in meta-analysis detected by a simple, graphical test. BMJ 315:629-634. doi:10.1136/bmj.315.7109.629

21. Crippa A, Orsini N (2016) Multivariate dose-response metaanalysis: the dosresmeta $r$ package. J Stat Softw 72:1-15

22. Viechtbauer W (2010) Conducting meta-analysis in R with the metafor package. J Stat Softw 36(3):1-48

23. Development Core Team R (2009) R: a language and environment for statistical computing. R Foundation for Statistical Computing, Vienna

24. Ronco AL, Mendilaharsu M, Boffetta P et al (2014) Meat consumption, animal products, and the risk of bladder cancer: a case-control study in Uruguayan men. Asian Pac J Cancer Prev APJCP 15:5805-5809

25. De Stefani E, Boffetta P, Ronco AL et al (2012) Processed meat consumption and risk of cancer: a multisite case-control study in Uruguay. Br J Cancer 107:1584-1588. doi:10.1038/bjc.2012.433

26. De Stefani E, Aune D, Boffetta P et al (2009) Salted meat consumption and the risk of cancer: a multisite case-control study in Uruguay. Asian Pac J Cancer Prev APJCP 10:853-857

27. De Stefani E, Boffetta P, Ronco AL et al (2008) Dietary patterns and risk of bladder cancer: a factor analysis in Uruguay. Cancer Causes Control CCC 19:1243-1249. doi:10.1007/ s10552-008-9195-9 
28. Lumbreras B, Garte S, Overvad K et al (2008) Meat intake and bladder cancer in a prospective study: a role for heterocyclic aromatic amines? Cancer Causes Control CCC 19:649-656. doi:10.1007/s10552-008-9121-1

29. Balbi JC, Larrinaga MT, De Stefani E et al (2001) Foods and risk of bladder cancer: a case-control study in Uruguay. Eur J Cancer Prev Off J Eur Cancer Prev Organ ECP 10:453-458

30. Radosavljević V, Janković S, Marinković J, Dokić M (2004) Non-occupational risk factors for bladder cancer: a case-control study. Tumori 90:175-180

31. Cross AJ, Leitzmann MF, Gail MH et al (2007) A prospective study of red and processed meat intake in relation to cancer risk. PLoS Med 4:e325. doi:10.1371/journal.pmed.0040325

32. Pou SA et al (2014) Dietary patterns and risk of urinary tract tumors: a multilevel analysis of individuals in rural and urban contexts. Eur J Nutr 53(5):1247-1253

33. Wilkens LR et al (1996) Risk factors for lower urinary tract cancer: the role of total fluid consumption, nitrites and nitrosamines, and selected foods. Cancer Epidemiol Biomark Prev 5(3):161-166

34. Chyou P-H, Nomura AMY, Stemmermann GN (1993) A prospective study of diet, smoking, and lower urinary tract cancer. Ann Epidemiol 3:211-216. doi:10.1016/1047-2797(93)90021-U

35. Radosavljević V, Janković S, Marinković J, Dokić M (2005) Diet and bladder cancer: a case-control study. Int Urol Nephrol 37:283-289. doi:10.1007/s11255-004-4710-8

36. Mills PK, Beeson WL, Phillips RL, Fraser GE (1991) Bladder cancer in a low risk population: results from the Adventist Health Study. Am J Epidemiol 133:230-239

37. Riboli E, González CA, López-Abente G et al (1991) Diet and bladder cancer in Spain: a multi-centre case-control study. Int J Cancer J Int Cancer 49:214-219

38. Baena AV, Allam MF, Del Castillo AS et al (2006) Urinary bladder cancer risk factors in men: a Spanish case-control study. Eur J Cancer Prev Off J Eur Cancer Prev Organ ECP 15:498-503. doi:10.1097/01.cej.0000215618.05757.04

39. Jakszyn P, González CA, Luján-Barroso L et al (2011) Red meat, dietary nitrosamines, and heme iron and risk of bladder cancer in the European Prospective Investigation into Cancer and Nutrition (EPIC). Cancer Epidemiol Biomark Prev Publ Am Assoc Cancer Res Cosponsored Am Soc Prev Oncol 20:555-559. doi:10.1158/1055-9965.EPI-10-0971

40. Ferrucci LM, Sinha R, Ward MH et al (2010) Meat and components of meat and the risk of bladder cancer in the NIH-AARP Diet and Health Study. Cancer 116:4345-4353. doi:10.1002/ cncr. 25463

41. Larsson SC, Johansson J-E, Andersson S-O, Wolk A (2009) Meat intake and bladder cancer risk in a Swedish prospective cohort. Cancer Causes Control CCC 20:35-40. doi:10.1007/ s10552-008-9214-x

42. Michaud DS, Holick CN, Giovannucci E, Stampfer MJ (2006) Meat intake and bladder cancer risk in 2 prospective cohort studies. Am J Clin Nutr 84:1177-1183
43. Nagano J, Kono S, Preston DL et al (2000) Bladder-cancer incidence in relation to vegetable and fruit consumption: a prospective study of atomic-bomb survivors. Int J Cancer 86:132-138. doi:10.1002/ (SICI)1097-0215(20000401)86:1<132:AID-IJC21>3.0.CO;2-M

44. Catsburg CE, Gago-Dominguez M, Yuan J-M et al (2014) Dietary sources of $\mathrm{N}$-nitroso compounds and bladder cancer risk: findings from the Los Angeles bladder cancer study. Int J Cancer 134:125-135. doi:10.1002/ijc.28331

45. Isa F, Xie L-P, Hu Z et al (2013) Dietary consumption and diet diversity and risk of developing bladder cancer: results from the South and East China case-control study. Cancer Causes Control CCC 24:885-895. doi:10.1007/s10552-013-0165-5

46. Wu JW, Cross AJ, Baris D et al (2012) Dietary intake of meat, fruits, vegetables, and selective micronutrients and risk of bladder cancer in the New England region of the United States. Br J Cancer 106:1891-1898. doi:10.1038/bjc.2012.187

47. Hu J, La Vecchia C, DesMeules M et al (2008) Meat and fish consumption and cancer in Canada. Nutr Cancer 60:313-324. doi:10.1080/01635580701759724

48. García-Closas R, García-Closas M, Kogevinas M et al (2007) Food, nutrient and heterocyclic amine intake and the risk of bladder cancer. Eur J Cancer Oxf Engl 43:1731-1740. doi:10.1016/j. ejca.2007.05.007

49. Tavani A, La Vecchia C, Gallus S et al (2000) Red meat intake and cancer risk: a study in Italy. Int J Cancer 86:425-428

50. World Cancer Research Fund/American Institute for Cancer Research (2011) Continuous Update Project Report. Food, nutrition, physical activity, and the prevention of colorectal cancer

51. Cancer IA for R on et al (2015) IARC Monographs evaluate consumption of red meat and processed meat. World Health Organ. Retrieved https://www.iarc.fr/en/media-centre/pr/2015/pdfs/ pr240_E.pdf

52. Pelucchi C, Bosetti C, Negri E et al (2006) Mechanisms of disease: the epidemiology of bladder cancer. Nat Clin Pract Urol 3:327-340. doi:10.1038/ncpuro0510

53. Bingham SA, Hughes R, Cross AJ (2002) Effect of white versus red meat on endogenous $\mathrm{N}$-nitrosation in the human colon and further evidence of a dose response. J Nutr 132:3522S-3525S

54. Bertram JS, Craig AW (1972) Specific induction of bladder cancer in mice by butyl-(4-hydroxybutyl)-nitrosamine and the effects of hormonal modifications on the sex difference in response. Eur J Cancer 8:587-594

55. Okada M, Ishidate M (1977) Metabolic fate of N-n-butyl-N(4-hydroxybutyl)-nitrosamine and its analogues. Selective induction of urinary bladder tumours in the rat. Xenobiotica Fate Foreign Compd Biol Syst 7:11-24

56. Skog KI, Johansson MA, Jägerstad MI (1998) Carcinogenic heterocyclic amines in model systems and cooked foods: a review on formation, occurrence and intake. Food Chem Toxicol Int J Publ Br Ind Biol Res Assoc 36:879-896

57. Phillips DH (1999) Polycyclic aromatic hydrocarbons in the diet. Mutat Res 443:139-147 